\title{
13-year nationwide cohort study of chronic kidney disease risk among treatment-naïve patients with chronic hepatitis B in Taiwan
}

\author{
Yi-Chun Chen ${ }^{1,2^{*}+}$, Yu-Chieh Su ${ }^{2,3}$, Chung-Yi Li $i^{4,5}$ and Shih-Kai Hung ${ }^{2,6^{*}+}$
}

\begin{abstract}
Background: Chronic hepatitis B virus (HBV) infection and chronic kidney disease (CKD) have high prevalences in Taiwan and worldwide. However, the association of untreated chronic hepatitis B virus (HBV) infection with chronic kidney disease (CKD) remains unclear.

Methods: This cohort study used claims data in the Taiwan National Health Insurance Research Database in 1996-2010, in which all diseases were classified by ICD-9-CM codes. We identified 17796 adults who had chronic HBV infection and did not take nucleos(t)ide analogues from 1998 to 2010 and also randomly selected 71184 matched controls without HBV in the same dataset. Cumulative incidences and adjusted hazard ratio (aHR) of incident CKD were evaluated through the end of 2010 after adjusting for competing mortality.

Results: The risk of CKD was significantly higher in the HBV cohort (13-year cumulative incidence, $6.2 \%$; 95 \% confidence interval [Cl], 5.4-7.1\%) than in the non-HBV cohort (2.7\%; $95 \% \mathrm{Cl}, 2.5-3.0 \%)(p<0.001)$, and the aHR was 2.58 (95\% Cl, 1.95-3.42; $p<0.001)$. Multivariable stratified analysis further verified significant associations of CKD with HBV in men of any age (aHR, 2.98; $95 \% \mathrm{Cl}, 2.32-3.83, p<0.001$ for men aged $<50$ years; aHR, 1.58; $95 \% \mathrm{Cl}$, $1.31-1.91, p<0.001$ for men aged $\geqq 50$ years) and women under the age of 50 (aHR, 2.99; $95 \% \mathrm{Cl}, 2.04-4.42$, $p<0.001$ ), but no significant association in women aged 50 or over.
\end{abstract}

Conclusion: Untreated chronic HBV infection is associated with increased risk of CKD. Hence, high-risk HBV-infected subjects should have targeted monitoring for the development of CKD.

Keywords: HBV infection, Chronic kidney disease, Cohort study, Taiwan National Health Insurance Research Database, Competing mortality

\section{Background}

Chronic kidney disease (CKD) is a global public health burden because of its increasing incidence and prevalence and progressive nature to end-stage renal disease (ESRD) [1]. Therefore, it is critical to identify risk factors for CKD to allow for effective surveillance. Beyond the traditional cardiovascular risk factors, infectious disease is an under-recognized risk factor for CKD [2].

\footnotetext{
* Correspondence: chenyichun0320@yahoo.com.tw; df254246@tzuchi.com.tw ${ }^{\dagger}$ Equal contributors

'Division of Nephrology, Department of Internal Medicine, Dalin Tzu Chi Hospital, Buddhist Tzu Chi Medical Foundation, Chiayi, No. 2, Minsheng Rd., Dalin Township, Chiayi County 622, Taiwan

${ }^{2}$ School of Medicine, Tzu Chi University, Hualien, Taiwan

Full list of author information is available at the end of the article
}

Hepatitis B affects approximately 350 million people worldwide [3], and is associated with high mortality and morbidity [4]. Apart from major liver complications, clinical evidence suggests that chronic HBV infection has a negative impact on renal function. HBV infection can lead to glomerulonephritis, even in the absence of cirrhosis [5]. A small controlled prospective study indicated that individual estimated glomerular filtration rate declined by approximately $-2 \mathrm{ml} / \mathrm{min} / \mathrm{y}$ in 60 untreated $\mathrm{HBV}$-infected patients without pre-existing renal disease, diabetes, or hypertension during the median follow-up of 24 months [6]. A 2-year cross-sectional HARPE study indicated that renal abnormalities were highly prevalent in treatment-naïve patients with chronic HBV infection [7]. A 2-year GLOBE study indicated that Telbivudine

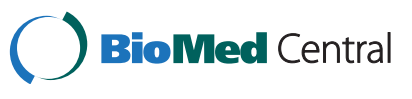


improved renal function in patients with chronic HBV infection [8]. Together, these findings provide a theoretical basis for further research of the negative impact of $\mathrm{HBV}$ infection on renal function.

Chronic HBV infection is associated with increased insulin resistance [9] and intensification of oxidative stress [10], and these may contribute to renal injury [11]. Therefore, it seems biologically plausible that HBVinfected subjects have increased risk for CKD. Our prior nationwide cohort study has demonstrated that untreated chronic HBV infection is associated with increased risk of ESRD [12]. However, four cross-sectional studies $[9,13-15]$ on the role of HBV in the development of CKD have had disparate results.

To date, there have been no cohort studies of the association of chronic HBV infection with risk of incident $\mathrm{CKD}$, and it is unclear which HBV-infected patients are more likely to develop CKD. This is a crucial issue, because the management of patients with coexisting HBV infection and CKD is a challenge [16] and the global burden of HBV infection and CKD is rising [17]. Taiwan has high prevalence of CKD and HBV infection, and thus provides an ideal setting for study of the relationship of both diseases. In the present study, we conducted a 13-year nationwide cohort study to examine whether untreated chronic HBV infection is associated with increased risk of incident CKD using reimbursement claims data from the Taiwan National Health Insurance Research Database (NHIRD).

\section{Methods \\ Database}

This nationwide cohort study used outpatient and inpatient claims from the NHIRD from 1996 to 2010, which is released by the National Health Research Institutes (NHRI) for Taiwan's National Health Insurance (NHI) Program. The NHI is a government-run, compulsoryenrolment, single-payer system that had a coverage rate of more than $99 \%$ by the end of 2010, and codes diagnoses according to ICD-9-CM (Additional file 1: Table S1). The NHIRD lacks information on laboratory and lifestyle data and severity of the disease condition. Our previous research provided details of the NHIRD [2, 12, 18-21]. In brief, the NHIRD has detailed healthcare data of 25.68 million enrollees (99.9 \% of the population) based on a random sample of all enrollees of the NHI program. There were no significant differences in age, sex, or healthcare costs between the sample group and all enrollees. The NHRI approved this study. Informed consent was not required because this is a secondary data analysis.

\section{Study cohorts}

Here, we used methods substantially similar to those we used in our earlier paper regarding the association of
HBV with ESRD [12], in which, briefly, we used the claims data from the NHIRD and identified 88790 study subjects from 1999 to 2010 . In the present study, we identified all adults with chronic HBV infection between 1 January 1998 and 31 December 2010 from the NHIRD. The index date was defined on the date of chronic HBV infection claim during the entry period. We excluded subjects who had HCV infection from 1996 to 2010 and CKD/ESRD from 1996 to the index date, or took nucleos(t)ide analogues (lamivudine, adefovir, entecavir, telbivudine) [12] before CKD event occurred. The NHI program in Taiwan was initiated in 1995, so the dataset only allows tracking of medical services from 1996 to 2010. In Taiwan, the NHI program has reimbursed nucleos(t)ide analogues for patients with chronic HBV infection since October 1, 2003 [22]. Thus, we could identify HBV patients using nucleos $(\mathrm{t})$ ide analogues in the NHIRD. There were 17796 untreated subjects with chronic HBV infection.

The non-HBV (control) cohort was extracted from the remaining subjects in the database. We first excluded subjects who had claim-based diagnoses of $\mathrm{HCV}$ or HBV infection from 1996 to 2010, or CKD/ESRD from 1996 to the index date. To consider the potential confounding by age, sex, and calendar year, we performed the individual matching technique in selecting controls. For each HBV case, we selected, by using simple random sampling method, 4 controls with the same gender and age category (18-39, 40-49, 50-59, $60-69$, or $\geqq 70$ years) as the HBV case. Additionally, the eligible controls must be actively insured for at least 1 day in the year when the HBV case was identified. We also managed to exclude the situation in which one control served multiple cases. By doing so, once a beneficiary was selected in a given year during the $\mathrm{HBV}$ patient recruitment period (i.e., 1998-2010), he/she was then eliminated from the pool of potential controls, which provide reassurance that no control subjects were repeatedly selected. Thus, for controls, the index date was within the same year of the index date of their matched cases. We used age stratification because previous research indicated an age-dependent association of HBV infection [4] and of CKD [19]. The control group ultimately included 71184 subjects.

\section{Definition of CKD}

The claims-based diagnosis of CKD was defined by the presence of 1 inpatient or 2 outpatient ICD-9-CM code 585 [23] in the claims and without catastrophic illness registration cards for ESRD (indicating the need for renal replacement therapy). The Taiwan Society of Nephrology launched a nationwide CKD Preventive Project covering CKD stages $1-5$ in 2004. The simplified Modification of Diet in Renal Disease equation along with proteinuria has been used to estimate the national 
prevalence of 5-stage CKD in Taiwan [1]. The ICD-9CM code 585 is consistent with the National Kidney Foundation's Kidney Disease Outcome Quality Initiative definition of CKD stages 1-5 [19], allowing for comparisons of the incidence and prevalence of CKD in Taiwan and the United States [24]. However, the CKD stage (severity) cannot be assessed in the NHIRD.

\section{Main outcome measurement}

Both cohorts were followed from the index date to the first diagnosis of CKD, death, or the end of 2010. Death is a competing event for any event of progression because patients who die during follow-up can no longer progress after death [25]. Therefore, death before reaching CKD, which could lead to informative censoring, was considered as a competing risk event in estimating the incidence of CKD. Death was defined by withdrawal from the NHI program [12].

\section{Potential confounders}

We considered several confounders associated with CKD [19], including major comorbidities between 1 January 1996 and the index date (diabetes, hypertension, coronary heart disease, hyperlipidemia, and cirrhosis), number of medical visits, urbanization level (urban, suburban, and rural) and enrollee category (EC), from EC1 (highest status) to EC4 (lowest status), as proxy measures of socioeconomic status. Previous Taiwanese studies reported an association of CKD with geographic region of residence $[19,26]$. Thus, geographic region of residence (northern, central, southern, or eastern Taiwan) was included to minimize potential confounding due to differences in accessibility and availability of medical care [18]. Herbs containing aristolochic acid, including (Guan) Mu Tong and (Guang) Fangchi, were associated with increased risk of CKD and could be identified from the NHIRD only before November 2003 [27]. Nevertheless, use of these herbs greater than 30 days from 1996 to endpoint outcome was also recorded. We also considered Deyo-Charlson Comorbidity Index (CCI) score and propensity score to control confounding and reduce bias in the background covariates between both cohorts in studies using healthcare administrative databases [28-30]. HBV status as the dependent variable and background covariates (including age, sex, major comorbidities, geographic region, urbanization level, EC, number of medial visits, and CCI score) as the independent variables were included in the calculation of propensity score using logistic regression. The propensity score model had a c-index of 0.66, indicating fair discrimination between the two cohorts [12].

\section{Statistical analyses}

We calculated and compared the cumulative incidence of CKD in data with competing risk by the modified
Kaplan-Meier method and Gray's method [31], and tested differences in the full time-to-event distributions between the study cohorts using modified log-rank test. We checked the interaction terms of HBV with sex, HBV with age, and HBV with age and sex, all of which were statistically significant. After confirming the assumption of proportional hazards, we applied the modified Cox proportional hazard model in the presence of competing mortality to examine the association of HBV with CKD [32], with adjustment for all covariates (age per year, sex, major comorbidities, use of herbs containing aristolochic acid, geographic region, urbanization level, EC, number of medical visits, CCI score, and propensity score) and three significant above-mentioned interaction terms. Next, we determined the influence of HBV on CKD according to sex and age in the presence of competing mortality. For this analysis, we classified patients as younger than 50 years or as 50 years and older, because previous research indicated that steatosis is a characteristic feature of chronic HBV infection [33] and that the biggest gender difference in steatosis prevalence was observed in individuals younger than 50 years [34]. As we simultaneously included comorbidities, social characteristics, $\mathrm{CCI}$ and propensity score in the multivariate regression model, a potential numerical problem concerned multicollinearity between covariates, which might render estimated regression coefficients invalid. We examined the potential multicollinearity among covariates and found that multicollinearity should not be a concern in our data as the multivariate Cox regression model had no large estimated slope coefficients and estimated standard error of the mean [35]. Besides, the tolerance level was greater than 0.1 for all covariates. We analyzed all data with SAS (version 9.2; SAS Institute, Inc., Cary, N.C.) and considered a twosided $p$-value less than 0.05 as statistically significant.

\section{Results}

\section{Baseline characteristics of the study cohorts}

The male-to-female ratio in the HBV cohort was 1.4. Compared with the non-HBV (control) cohort, the HBV cohort was more likely to have comorbidities including diabetes, hypertension, hyperlipidemia, and cirrhosis, as well as frequent use of herbs containing aristolochic acid, higher socioeconomic status (EC 1 and 2), more medical visits, higher propensity score and CCI score, and to reside in urban areas and northern, central, and eastern regions; all with considerable significance (Table 1).

\section{Cumulative incidences of CKD between the HBV and control cohorts in the presence of competing mortality}

The 1-, 3-, 5-, 7-, 9-, 11-, and 13-year cumulative incidences of CKD were $0.26 \%$ vs. $0.10 \%, 0.77 \%$ vs. $0.33 \%$, $1.35 \%$ vs. $0.65 \%, 2.00 \%$ vs. $1.00 \%, 2.90 \%$ vs. $1.48 \%$, 
Table 1 Sociodemographic characteristics and comorbidities between cohorts with and without hepatatis B virus (HBV) infection in Taiwan, 1998-2010 $(n=88980)$

\begin{tabular}{|c|c|c|c|}
\hline & $\begin{array}{l}\text { HBV } \\
\text { cohort }\end{array}$ & $\begin{array}{l}\text { Non-HBV } \\
\text { cohort }\end{array}$ & \\
\hline Variables & $\begin{array}{l}(n=17796) \\
N(\%)\end{array}$ & $\begin{array}{l}(n=71184) \\
\mathrm{N}(\%)\end{array}$ & $P$ \\
\hline Sex & & & 1.00 \\
\hline Men & $\begin{array}{l}10428 \\
(58.6)\end{array}$ & $41712(58.6)$ & \\
\hline Women & 7368 (41.4) & $29472(41.4)$ & \\
\hline Age (year) & & & 1.00 \\
\hline $18-39$ & $8514(47.8)$ & $34056(47.8)$ & \\
\hline $40-49$ & 4914 (27.6) & $19656(27.6)$ & \\
\hline $50-59$ & $2857(16.1)$ & $11428(16.1)$ & \\
\hline $60-69$ & $1146(6.4)$ & $4584(6.4)$ & \\
\hline$\geqq 70$ & $365(2.1)$ & $1460(2.1)$ & \\
\hline \multicolumn{4}{|l|}{ Major comorbidities } \\
\hline Diabetes & $1660(9.3)$ & $5181(7.3)$ & $<0.001$ \\
\hline Hypertension & $2511(14.1)$ & $9020(12.7)$ & $<0.001$ \\
\hline Coronary heart disease & $1039(5.8)$ & $4392(6.2)$ & 0.10 \\
\hline Hyperlipidemia & $2484(14.0)$ & $66.71(9.4)$ & $<0.001$ \\
\hline Cirrhosis & $638(3.6)$ & $466(0.7)$ & $<0.001$ \\
\hline $\begin{array}{l}\text { Use of herbs containing } \\
\text { aristolochic acid }\end{array}$ & $365(2.1)$ & $931(1.3)$ & $<0.001$ \\
\hline \multicolumn{4}{|l|}{ Geographic region } \\
\hline Northern & 8967 (50.4) & $35017(49.2)$ & \\
\hline Central & 4191 (23.6) & $16280(22.9)$ & \\
\hline Eastern & $434(2.4)$ & $1588(2.2)$ & \\
\hline Southern & 4204 (23.6) & $18299(25.7)$ & \\
\hline Urbanization level & & & 0.001 \\
\hline Urban & 5939 (33.4) & $22745(32.0)$ & \\
\hline Suburban & $8216(46.2)$ & $33438(47.0)$ & \\
\hline Rural & $3640(20.4)$ & $15002(21.0)$ & \\
\hline \multicolumn{4}{|l|}{ Enrollee category } \\
\hline 1 & $9420(52.9)$ & $35633(50.0)$ & \\
\hline 2 & $546(3.1)$ & $1830(2.6)$ & \\
\hline 3 & $5297(29.8)$ & $22157(31.1)$ & \\
\hline 4 & $2533(14.2)$ & $11564(16.3)$ & \\
\hline No. of medical visits (mean \pm SD) & $17.7 \pm 15.1$ & $15.8 \pm 15.3$ & $<0.001$ \\
\hline Propensity score (mean \pm SD) & $0.23 \pm 0.09$ & $0.19 \pm 0.07$ & $<0.001$ \\
\hline $\begin{array}{l}\text { Charlson Comorbiditv Index } \\
\text { score (mean } \pm \text { SD) }\end{array}$ & $1.1 \pm 1.2$ & $0.6 \pm 1.2$ & $<0.001$ \\
\hline
\end{tabular}

Categorical variables given as number (percentage); continuous variable, as mean \pm standard deviation

$4.30 \%$ vs. $2.12 \%$, and $6.20 \%$ vs. $2.74 \%$, respectively, in the HBV cohort compared with the control cohort (all $p<0.001$, Table 2). Therefore, the risk of CKD was significantly higher in the HBV cohort (13-year cumulative
Table 2 Cumulative incidences of chronic kidney disease (CKD) between the HBV and non-HBV cohorts in the presence of competing mortality

\begin{tabular}{llll}
\hline & HBV cohort, $(95 \% \mathrm{Cl})$ & Non-HBV cohort, (95 \% Cl) & $P^{*}$ \\
\hline CKD event & & & \\
At 1 year & $0.26 \%(0.19-0.35 \%)$ & $0.10 \%(0.08-0.12 \%)$ & $<0.001$ \\
At 3 years & $0.77 \%(0.64-0.92 \%)$ & $0.33 \%(0.29-0.38 \%)$ & $<0.001$ \\
At 5 years & $1.35 \%(1.17-1.55 \%)$ & $0.65 \%(0.59-0.72 \%)$ & $<0.001$ \\
At 7 years & $2.00 \%(1.76-2.26 \%)$ & $1.00 \%(0.91-1.09 \%)$ & $<0.001$ \\
At 9 year & $2.90 \%(2.58-3.24 \%)$ & $1.48 \%(1.37-1.60 \%)$ & $<0.001$ \\
At 11 year & $4.30 \%(3.85-4.79 \%)$ & $2.12 \%(1.96-2.29 \%)$ & $<0.001$ \\
At 13 year $\quad 6.20 \%(5.39-7.08 \%)$ & $2.74 \%(2.49-3.01 \%)$ & $<0.001$ \\
\hline
\end{tabular}

*Two sample proportion test. Abbreviations: $\mathrm{Cl}$ confidence interval

incidence, $6.2 \%$; $95 \% \mathrm{CI}, 5.4-7.1 \%)$ than the control cohort (2.7 \%; 95 \% CI, $2.5-3.0 \%)(p<0.001$, Fig. 1$)$.

\section{Chronic HBV infection as an independent risk factor for CKD}

The mean duration of follow-up was 6.55 and 6.56 years for the HBV and control cohorts, respectively (Additional file 2: Table S2). Among the 88980 subjects, 1247 subjects (1.4\%) developed CKD during the study period, with 425 (2.4\%) and 822 (1.2\%) from the HBV and control cohorts, respectively. The modified multivariableadjusted Cox proportional hazard model in the presence of competing mortality revealed that CKD was independently associated with HBV (adjusted hazard ratio [aHR], 2.58 ; $95 \%$ confidence interval [CI], 1.95-3.42; $p<0.001$ ), men $(1.38 ; 1.11-1.73 ; p=0.004)$; age $(1.06 ; 1.05-1.07$; $p<0.001)$, diabetes $(2.41 ; 1.94-2.99 ; p<0.001)$, hypertension $(1.92 ; 1.64-2.24 ; p<0.001)$, residence in central (1.31; $1.12-1.53 ; p<0.001)$, eastern $(1.49 ; 1.06-2.09 ; p=0.023)$, southern $(1.21 ; 1.04-1.42 ; p=0.016)$ Taiwan, and the number of medical visits $(1.01 ; 1.009-1.014 ; p<0.001)$ (Table 3).

We further performed a sensitivity analysis to test the robustness of these results. We excluded diabetic or cirrhotic subjects from both cohorts at baseline, also indicating a positive association of HBV with CKD (2.61, 1.97-3.46; 2.58, 2.04-3.99; respectively) (data not shown).

\section{Association of CKD with HBV by sex and age}

After adjusting for competing mortality, multivariable stratified analysis further verified significant associations of CKD risk with HBV in men (adjusted HR, 3.05; $95 \%$ CI, $2.50-3.71 ; p<0.001)$, women $(2.56 ; 1.93-3.41 ; p<$ $0.001)$, subjects younger than 50 years $(2.86 ; 1.98-4.13$; $p<0.001)$, men younger $(2.98 ; 2.32-3.83 ; p<0.001)$ and older than 50 years $(1.58 ; 1.31-1.91 ; p<0.001)$, and women younger than 50 years $(2.99 ; 2.04-4.42 ; p<$ 0.001 ), and no significant association in subjects older than 50 years $(1.19 ; 0.90-1.56 ; p=0.22)$ and women older than 50 years $(1.18 ; 0.90-1.55 ; p=0.24)$ (Table 4$)$. 


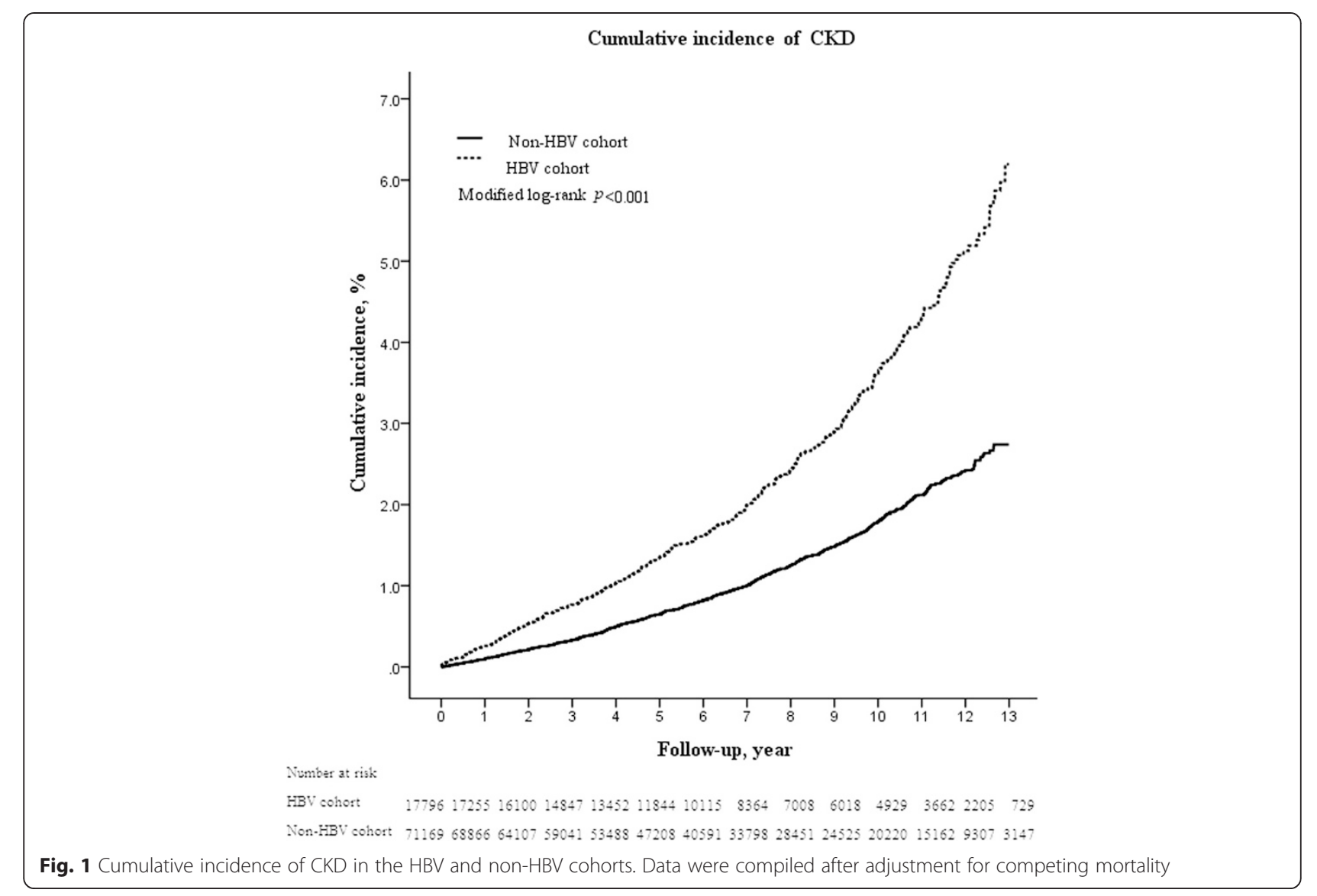

\section{Discussion}

To our knowledge, this is the first large nationwide cohort study to demonstrate an increased risk for CKD in untreated subjects with chronic HBV infection and simultaneously to determine the overall risk and age- and sex-specific risks of CKD. In this study, CKD was 2.58fold more likely in HBV-infected subjects, relative to controls, after controlling for potential confounders and competing mortality. We also found that the risk of CKD in HBV-infected subjects was somewhat similar (range from 2 to 3 ) in men of any age and women under the age of 50. Noteworthily, HBV was not associated with CKD risk in women aged 50 or over. This information has important clinical implications for the design of surveillance programs that assess chronic HBV infection and CKD.

The association between chronic HBV infection and the development of CKD remains controversial. Two cross-sectional studies $[7,15]$ demonstrated a positive association of $\mathrm{HBV}$ with CKD. A 2-year multicentric cross-sectional French study reported that CKD was highly prevalent in 268 treatment-native patients with chronic HBV infection [7]. A cross-sectional Taiwanese study [15] reported that HBV was associated with CKD in 416 HBV-infected adults; however, another cross- sectional Taiwanese study by the same research group reported that HBV was not associated with CKD or proteinuria in 5424 HBV-infected adults [14]. Similarly, a cross-sectional study of $328 \mathrm{HBV}$-infected adults in Beijing [13] reported that HBV was not associated with CKD or albuminuria. On the other hand, a crosssectional Japanese study of 130 HBV-infected adults [9] showed that HBV was not associated with albuminuria, but was inversely associated with CKD. The discrepancies among these studies may be attributed to the crosssectional nature of their design, use of small sample sizes, and differences among the specific populations. Moreover, cross-sectional analyses cannot establish whether HBV infection preceded development of CKD. The present study was cohort design and used a large dataset, which afforded considerable statistical power and allowed tracking of incident CKD events over 13 years. We demonstrated an increased risk of CKD in untreated patients with chronic HBV infection that was independent of the presence of diabetes or cirrhosis.

Our HBV cohort tended to be younger and male, and to have a higher socioeconomic status, consistent with our prior research [12]. On stratified analysis, we found significant associations of HBV with CKD risk in men of any age (especially under the age of 50) and women 
Table 3 Crude and adjusted hazard ratios (HR) for chronic kidney disease (CKD), with adjustment for competing morality

\begin{tabular}{|c|c|c|c|c|c|c|}
\hline \multirow[t]{2}{*}{ Variable } & \multicolumn{3}{|c|}{ Crude } & \multicolumn{3}{|c|}{ Adjusted $^{*}$} \\
\hline & $\mathrm{HR}$ & $(95 \% \mathrm{Cl})$ & $P$ & $\mathrm{HR}$ & $(95 \% \mathrm{Cl})$ & $P$ \\
\hline HBV (Yes/No) & 2.08 & $1.85-2.34$ & $<0.001$ & 2.58 & $1.95-3.42$ & $<0.001$ \\
\hline Sex (Men/Women) & 1.32 & $1.18-1.49$ & $<0.001$ & 1.38 & $1.11-1.73$ & 0.004 \\
\hline Age (per year) & 1.07 & $1.06-1.07$ & $<0.001$ & 1.06 & $1.05-1.07$ & $<0.001$ \\
\hline \multicolumn{7}{|l|}{ Major comorbidities (Yes/No) } \\
\hline Diabetes & 5.30 & $4.69-5.98$ & $<0.001$ & 2.41 & $1.94-2.99$ & $<0.001$ \\
\hline Hypertension & 5.00 & $4.47-5.60$ & $<0.001$ & 1.92 & $1.64-2.24$ & $<0.001$ \\
\hline Coronary heart disease & 2.88 & $2.47-3.37$ & $<0.001$ & 0.90 & $0.66-1.23$ & 0.51 \\
\hline Hyperlipidemia & 2.77 & $2.41-3.18$ & $<0.001$ & 1.19 & $0.95-1.49$ & 0.13 \\
\hline Cirrhosis & 2.29 & $1.67-3.15$ & $<0.001$ & 1.00 & $0.33-3.02$ & 0.10 \\
\hline Use of herbs containing aristolochic acid (Yes/No) & 1.13 & $0.75-1.70$ & 0.57 & 0.88 & $0.59-1.33$ & 0.55 \\
\hline \multicolumn{7}{|l|}{ Geographic region } \\
\hline Northern & 1.00 & Reference & & 1.00 & Reference & \\
\hline Central & 1.22 & $1.08-1.39$ & 0.002 & 1.31 & $1.12-1.53$ & $<0.001$ \\
\hline Eastern & 1.40 & $1.03-1.92$ & 0.034 & 1.49 & $1.06-2.09$ & 0.023 \\
\hline Southern & 1.19 & $1.05-1.34$ & 0.006 & 1.21 & $1.04-1.42$ & 0.016 \\
\hline \multicolumn{7}{|l|}{ Urbanization level } \\
\hline Urban & 1.00 & Reference & & 1.00 & Reference & \\
\hline Suburban & 0.94 & $0.84-1.05$ & 0.27 & 0.98 & $0.85-1.14$ & 0.82 \\
\hline Rural & 1.39 & $1.23-1.58$ & $<0.001$ & 0.92 & $0.77-1.11$ & 0.38 \\
\hline \multicolumn{7}{|l|}{ Enrollee category } \\
\hline 1 & 1.00 & Reference & & 1.00 & Reference & \\
\hline 2 & 0.55 & $0.34-0.87$ & 0.011 & 0.86 & $0.53-1.38$ & 0.53 \\
\hline 3 & 1.52 & $1.36-1.70$ & $<0.001$ & 1.14 & $0.97-1.35$ & 0.11 \\
\hline 4 & 1.45 & $1.27-1.66$ & $<0.001$ & 1.12 & $0.91-1.38$ & 0.28 \\
\hline No. of medical visits & 1.02 & $1.01-1.02$ & $<0.001$ & 1.01 & $1.009-1.014$ & $<0.001$ \\
\hline Charlson Comorbidity Index score & 1.31 & $1.29-1.34$ & $<0.001$ & 0.97 & $0.81-1.14$ & 0.68 \\
\hline Propensity score & 1.42 & $1.36-1.47$ & $<0.001$ & 1.10 & $0.77-1.57$ & 0.60 \\
\hline
\end{tabular}

Abbreviations: $\mathrm{HBV}$ hepatitis B virus, $\mathrm{Cl}$ confidence interval

*Adjusted for all covariates (age per year, sex, major comorbidities, use of herbs containing aristolochic acid, geographic region, urbanization level, enrollee category, number of medical visits, Charlson Comorbidity Index score, and propensity score), and interaction terms (HBV/age, HBV/sex, and HBV/age/sex)

under the age of 50, similar to our prior research [12]. Previous research reported that men have increased risk for development of CKD [19], and that men have a lower clearance rate than females, so are therefore more susceptible to development of chronic HBV infection [36]. Steatosis, a characteristic feature of chronic HBV infection, can increase lipid peroxidation and plasma inflammatory biomarkers [33], and these may contribute to endothelial dysfunction and renal injury [11]. The biggest sex difference in steatosis occurs in subjects younger than 50 years [33]. Taken together, these results may explain our observations regarding the effect of age and gender on the risk for development of CKD following untreated chronic HBV infection.

The pathogenesis of HBV-mediated renal injury probably depends on interactions of the virus with the host and various environmental factors [3]. Our results indicated that CKD ensued following untreated chronic HBV infection during the mean follow-up of 5.7 years, the interval of which was similar to 60 months in a case series [5]. HBV-related renal injury is believed to be due to the deposition of immune complexes of HBV antigens and host antibodies [3]. Other research also explored the possible effect of HBV on the development of nephropathy. HBV antigens (HBsAg, $\mathrm{HBcAg}$, and $\mathrm{HBeAg}$ ) and HBV DNA have been detected in glomeruli [37] and tubular epithelia [38], respectively. HBV DNA negative or positive sera of patients with chronic HBV infection promoted apoptotic damage in renal tubular cells via up-regulation of Fas gene expression, and these patients also had a higher circulating level of transforming growth factor- $\beta$, which is implicated in the potentiation 
Table 4 Association of chronic kidney disease (CKD) with HBV, stratified by sex and age, with adjustment for competing mortality

\begin{tabular}{|c|c|c|c|c|c|c|c|c|c|c|}
\hline & \multicolumn{3}{|c|}{ HBV cohort } & \multicolumn{3}{|c|}{ Non-HBV cohort } & \multirow[b]{2}{*}{ Crude } & \multicolumn{3}{|c|}{ Hazard ratio $(95 \%$ Cl) } \\
\hline & $\bar{n}$ & PY & $\overline{C K D}$ events & $\bar{n}$ & PY & $\overline{C K D}$ events & & $\bar{P}$ & Adjusted $^{*}$ & $P$ \\
\hline \multicolumn{11}{|l|}{ Sex } \\
\hline Men & 10428 & 68249 & 294 & 41712 & 274693 & 525 & $2.26(1.96-2.61)$ & $<0.001$ & $3.05(2.50-3.71) \dagger$ & $<0.001$ \\
\hline Women & 7368 & 48243 & 131 & 29472 & 192357 & 297 & $1.77(1.44-2.18)$ & $<0.001$ & $2.56(1.93-3.41) \dagger$ & $<0.001$ \\
\hline \multicolumn{11}{|l|}{ Age (year) } \\
\hline$<50$ & 13428 & 84358 & 203 & 53712 & 337813 & 191 & $4.30(3.53-5.24)$ & $<0.001$ & $2.86(1.98-4.13)$ & $<0.001$ \\
\hline$\geqq 50$ & 4368 & 32133 & 222 & 17472 & 129237 & 631 & $1.40(1.20-1.63)$ & $<0.001$ & $1.19(0.90-1.56)$ & 0.22 \\
\hline \multicolumn{11}{|l|}{ Men } \\
\hline$<50$ years & 7888 & 49981 & 140 & 31552 & 200858 & 132 & $4.28(3.38-5.43)$ & $<0.001$ & $2.98(2.32-3.83)$ & $<0.001$ \\
\hline$\geqq 50$ years & 2540 & 18268 & 154 & 10160 & 73835 & 393 & $1.57(1.30-1.89)$ & $<0.001$ & $1.58(1.31-1.91)$ & $<0.001$ \\
\hline \multicolumn{11}{|l|}{ Women } \\
\hline$<50$ years & 5540 & 34378 & 63 & 22160 & 136955 & 59 & $4.34(3.05-6.19)$ & $<0.001$ & $2.99(2.04-4.42)$ & $<0.001$ \\
\hline$\geqq 50$ years & 1828 & 13865 & 68 & 7312 & 55402 & 238 & $1.13(0.86-1.48)$ & 0.37 & $1.18(0.90-1.55)$ & 0.24 \\
\hline
\end{tabular}

Abbreviations: $\mathrm{HBV}$ hepatitis $\mathrm{B}$ virus, $\mathrm{PY}$ person-year, $\mathrm{Cl}$ confidence interval

${ }^{*}$ Adjusted for all covariates (age, sex, major comorbidities, use of herbs containing aristolochic acid, geographic region, urbanization level, enrollee category, number of medical visits, Charlson Comorbidity Index score, and propensity score) and interaction terms (HBV/age, HBV/sex, HBV/age/sex), minus the covariate and interaction terms on which stratified

†Age was treated as a continuous variable in multivariate analysis

of apoptosis and renal fibrosis [39]. HBV is associated with increased insulin resistance [9] and oxidative stress [10], and these are implicated in the progression of nephropathy [11]. However, only a small number of the 350 million HBV carriers develop glomerulonephritis [4]. This indicates that chronic HBV infection by itself is insufficient for development of nephropathy, and that additional socio-environmental conditions, alterations in cell-mediated immunity, genetic susceptibility, and other factors are also important [3]. This may explain the lower 13-year cumulative incidence rate (6.2 \%) of CKD following untreated chronic HBV infection in this study. However, the HBV-positive population is increasing by 50 million per year, despite a marked increase in vaccination rates [40]. Therefore, it is conceivable that the burden of CKD following chronic HBV infection is increasing.

The major strength of our study is that it was designed to reduce selection bias (due to use of a large nationwide population-based and highly representative sample with random sampling), environmental effects (due to the availability of socioeconomic indicators for all subjects) [18], and detection bias (due to consideration of complete histories of the use of medical services) [19]. In addition, the study population was well defined and follow-up was complete because our design relied on computerized registries that provided complete nationwide coverage. Therefore, our finding of increased risk of CKD in HBV-infected subjects is robust.

Some potential limitations should be noted. First, misclassification of diseases may occur when an administration database is used. However, the NHI Administration established an audit and penalty system for quality monitoring to ensure accuracy of claims and minimize misclassification error [41]. Moreover, both CKD and viral hepatitis are important health problems in Taiwan, so the government has strict guidelines for diagnosis [42]. Second, some of our control subjects may have had subclinical HBV infection. However, if $\mathrm{HBV}$ is associated with CKD, this misclassification would lead to lower HRs, thus supporting the robustness of our findings. Third, although nucleos $(\mathrm{t})$ ide analogues for HBV patients have been covered under the NHI program since October 1, 2003, this reimbursement requires patients to fulfill certain criteria, such as two-fold increase or more in serum alanine aminotransferase level and HBV DNA titer greater than $2000 \mathrm{IU} / \mathrm{mL}$ [43]. Some HBV patients may use self-paid nucleos(t)ide analogues before October 1, 2003 or when they do not fulfill reimbursed criteria, and thus may be inappropriately classified into the untreated cohort. These potential misclassifications may lead to an underestimation of the association [22]. Fourth, the NHIRD lacks information on family history of kidney diseases, lifestyle, body weight, and laboratory data (e.g., levels of serum HBV DNA and HBV genotype), which may contribute to CKD risk. Thus, this may lead to the difference of propensity score between both cohorts. Nevertheless, we added $\mathrm{CCI}$ score into the propensity analysis in order to reach the comparability of both cohorts and included CCI score and propensity score in multivariable and stratified analyses to control confounding in healthcare administrative databases $[12,28,29]$. Although unmeasured confounders my still exist as with any observational study, we believe the methodology used in the present study is solid and robust. 


\section{Conclusions}

This large national cohort study indicates that untreated chronic HBV infection is associated with increased risk of CKD, especially males and younger age. High-risk HBV-infected subjects should have targeted monitoring for the development of CKD.

\section{Additional files}

Additional file 1: Table S1. ICD-9-CM codes in the present study. Additional file 2: Table S2. Chronic kidney disease (CKD) occurrence over a 13-year follow-up.

\section{Abbreviations}

CKD: Chronic kidney disease; HBV: Hepatitis B virus; HR: Hazard ratio; Cl: Confidence interval; ESRD: End-stage renal disease; NHIRD: National Health Insurance Research Database; NHRI: National Health Research Institutes; NHI: National Health Insurance; EC: Enrollee category; CCl: Charlson Comorbidity Index.

\section{Competing interests}

The authors declare that they have no competing interests.

\section{Authors' contributions}

Conceived and designed the experiments: YCC. Contributed reagents/ materials/analysis tools: SKH, YCS. Performed the experiments: YCC. Analyzed the data: YCC, SKH, YCS. Wrote the paper: YCC. Provided constructive opinions and suggestions: YCC, SKH, YCS, CYL. All authors read and approved the final manuscript.

\section{Acknowledgements}

This study is based partly on secondary data from the National Health Insurance Research Database provided by the National Health Insurance Administration, Ministry of Health and Welfare, Taiwan. The interpretations and conclusions herein do not represent the views of the National Health Insurance Administration, Ministry of Health and Welfare, or National Health Research Institutes.

\section{Author details}

'Division of Nephrology, Department of Internal Medicine, Dalin Tzu Chi Hospital, Buddhist Tzu Chi Medical Foundation, Chiayi, No. 2, Minsheng Rd., Dalin Township, Chiayi County 622, Taiwan. ${ }^{2}$ School of Medicine, Tzu Chi University, Hualien, Taiwan. ${ }^{3}$ Division of Hematology-Oncology, Department of Internal Medicine, Dalin Tzu Chi Hospital, Buddhist Tzu Chi Medical Foundation, Chiayi, Taiwan. ${ }^{4}$ Department and Graduate Institute of Public Health, College of Medicine, National Cheng Hung University, Tainan, Taiwan. ${ }^{5}$ Department of Public Health, College of Public Health, China Medical University, Taichung, Taiwan. ${ }^{6}$ Department of Radiation Oncology, Dalin Tzu Chi Hospital, Buddhist Tzu Chi Medical Foundation, Chiayi, Taiwan.

Received: 6 March 2015 Accepted: 2 July 2015

Published online: 22 July 2015

\section{References}

1. Wen CP, Cheng TY, Tsai MK, Chang YC, Chan HT, Tsai SP, et al. All-cause mortality attributable to chronic kidney disease: a prospective cohort study based on 462293 adults in Taiwan. Lancet. 2008;371:2173-82.

2. Chen YC, Chiou WY, Hung SK, Su YC, Hwang SJ. Hepatitis C virus itself is a causal risk factor for chronic kidney disease beyond traditional risk factors: a 6-year nationwide cohort study across Taiwan. BMC Nephrol. 2013;14:187.

3. Bhimma R, Coovadia HM. Hepatitis B virus-associated nephropathy. Am J Nephrol. 2004;24:198-211.

4. Levy M, Chen N. Worldwide perspective of hepatitis B-associated glomerulonephritis in the 80s. Kidney Int Suppl. 1991;35:S24-33.

5. Lai KN, Li PK, Lui SF, Au TC, Tam JS, Tong KL, et al. Membranous nephropathy related to hepatitis B virus in adults. N Engl J Med. 1991;324:1457-63.
6. Mauss S, Berger F, Filmann N, Hueppe D, Henke J, Hegener P, et al. Effect of HBV polymerase inhibitors on renal function in patients with chronic hepatitis B. J Hepatol. 2011;55:1235-40.

7. Amet S, Bronowicki JP, Thabut D, Zoulim F, Bourliere M, Mathurin P, et al Prevalence of renal abnormalities in chronic HBV infection: The HARPE study. Liver Int. 2015;35:148-55.

8. Gane EJ, Deray G, Liaw YF, Lim SG, Lai CL, Rasenack J, et al. Telbivudine improves renal function in patients with chronic hepatitis B. Gastroenterology. 2014;146:138-46.

9. Ishizaka N, Ishizaka Y, Seki G, Nagai R, Yamakado M, Koike K. Association between hepatitis $\mathrm{B} / \mathrm{C}$ viral infection, chronic kidney disease and insulin resistance in individuals undergoing general health screening. Hepatol Res. 2008:38:775-83.

10. Pawlak K, Pawlak D, Mysliwiec M. Hepatitis intensified oxidative stress, MIP1 beta and RANTES plasma levels in uraemic patients. Cytokine. 2004;28:197-204

11. Sarafidis PA, Ruilope LM. Insulin resistance, hyperinsulinemia, and renal injury: mechanisms and implications. Am J Nephrol. 2006;26:232-44.

12. Chen YC, Su YC, Li CY, Wu CP, Lee MS. A nationwide cohort study suggests chronic hepatitis $B$ virus infection increases the risk of end-stage renal disease among patients in Taiwan. Kidney Int. 2015;87:1030-8.

13. Cai J, Fan X, Mou L, Gao B, Liu X, Li J, et al. Association of reduced renal function with hepatitis $B$ virus infection and elevated alanine aminotransferase. Clin J Am Soc Nephrol. 2012;7:1561-6.

14. Lee JJ, Lin MY, Yang YH, Lu SN, Chen HC, Hwang SJ. Association of hepatitis $C$ and $B$ virus infection with CKD in an endemic area in Taiwan: a crosssectional study. Am J Kidney Dis. 2010;56:23-31.

15. Lin MY, Chiu YW, Lee $\mathrm{CH}$, Yu HY, Chen HC, Wu MT, et al. Factors associated with CKD in the elderly and nonelderly population. Clin J Am Soc Nephrol. 2013;8:33-40.

16. Unger JK, Peters $\mathrm{H}$. Hepatitis B in chronic kidney disease: moving toward effective prevention. Kidney Int. 2008;73:799-801.

17. Chacko EC, Surrun SK, Mubarack Sani TP, Pappachan JM. Chronic viral hepatitis and chronic kidney disease. Postgrad Med J. 2010;86:486-92.

18. Sun Y, Chang YH, Chen HF, Su YH, Su HF, Li CY. Risk of Parkinson disease onset in patients with diabetes: a 9-year population-based cohort study with age and sex stratifications. Diabetes Care. 2012;35:1047-9.

19. Chen YC, Lin HY, Li CY, Lee MS, Su YC. A nationwide cohort study suggests that hepatitis $C$ virus infection is associated with increased risk of chronic kidney disease. Kidney Int. 2014;85:1200-7.

20. Chen HF, Chen P, Li CY. Risk of malignant neoplasms of liver and biliary tract in diabetic patients with different age and sex stratifications. Hepatology. 2010;52:155-63.

21. Chen YC, Su YC, Lee CC, Huang YS, Hwang SJ. Chronic kidney disease itself is a causal risk factor for stroke beyond traditional cardiovascular risk factors: a nationwide cohort study in Taiwan. PLoS One. 2012;7, e36332.

22. Wu CY, Chen YJ, Ho HJ, Hsu YC, Kuo KN, Wu MS, et al. Association between nucleoside analogues and risk of hepatitis B virus-related hepatocellular carcinoma recurrence following liver resection. JAMA. 2012;308:1906-14.

23. Collins AJ, Foley RN, Herzog C, Chavers B, Gilbertson D, Ishani A, et al. United States Renal Data System 2008 Annual Data Report. Am J Kidney Dis. 2009;53:S1-374.

24. Collins AJ, Foley RN, Herzog C, Chavers B, Gilbertson D, Ishani A, et al. US Renal Data System 2010 Annual Data Report. Am J Kidney Dis. 2011;57(A8):e1-526.

25. Boucquemont J, Heinze G, Jager KJ, Oberbauer R, Leffondre K. Regression methods for investigating risk factors of chronic kidney disease outcomes: the state of the art. BMC Nephrol. 2014;15:45.

26. Kuo HW, Tsai SS, Tiao MM, Yang CY. Epidemiological features of CKD in Taiwan. Am J Kidney Dis. 2007:49:46-55.

27. Lai MN, Lai JN, Chen PC, Tseng WL, Chen YY, Hwang JS, et al. Increased risks of chronic kidney disease associated with prescribed Chinese herbal products suspected to contain aristolochic acid. Nephrology (Carlton). 2009;14:227-34.

28. Patorno E, Grotta A, Bellocco R, Schneeweiss S. Propensity score methodology for confounding control in health care utilization databases. Epidemiol Biostat Public Health. 2013;10, e8940.

29. Schneeweiss S, Maclure M. Use of comorbidity scores for control of confounding in studies using administrative databases. Int J Epidemiol. 2000;29:891-8

30. D'Agostino Jr RB. Propensity scores in cardiovascular research. Circulation. 2007;115:2340-3. 
31. Gray RJ. A class of K-sample tests for comparing the cumulative incidence of a competing risk. Ann Stat. 1988;16:1141-54.

32. Fine JP, Gray RJ. A proportional hazards model for the subdistribution of a competing risk. J Am Stat Assoc. 1999;94:496-509.

33. Shimizu I, Kohno N, Tamaki K, Shono M, Huang HW, He JH, et al. Female hepatology: favorable role of estrogen in chronic liver disease with hepatitis B virus infection. World J Gastroenterol. 2007;13:4295-305.

34. Weston SR, Leyden W, Murphy R, Bass NM, Bell BP, Manos MM, et al. Racial and ethnic distribution of nonalcoholic fatty liver in persons with newly diagnosed chronic liver disease. Hepatology. 2005;41:372-9.

35. Harrell Jr FE, Lee KL, Pollock BG. Regression models in clinical studies: determining relationships between predictors and response. J Natl Cancer Inst. 1988:80:1198-202.

36. London WT, Drew JS. Sex differences in response to hepatitis B infection among patients receiving chronic dialysis treatment. Proc Natl Acad Sci U S A. 1997;74:2561-3.

37. Lai KN, Lai FM. Clinical features and the natural course of hepatitis B virusrelated glomerulopathy in adults. Kidney Int Suppl. 1991;35:S40-4.

38. Lai KN, Ho RT, Tam JS, Lai FM. Detection of hepatitis B virus DNA and RNA in kidneys of HBV related glomerulonephritis. Kidney Int. 1996;50:1965-77.

39. Deng CL, Song XW, Liang HJ, Feng C, Sheng YJ, Wang MY. Chronic hepatitis B serum promotes apoptotic damage in human renal tubular cells. World J Gastroenterol. 2006;12:1752-6.

40. Patton $\mathrm{H}$, Tran $\mathrm{T}$. Management of hepatitis B during pregnancy. Nat Rev Gastroenterol Hepatol. 2014;11:402-9.

41. Sheu JJ, Kang JH, Lin HC. Hyperthyroidism and risk of ischemic stroke in young adults: a 5-year follow-up study. Stroke. 2010;41:961-6.

42. National Health Insurance Administration, Ministry of Health and Welfare, Taiwan. http://www.nhi.gov.tw. Accessed June 1, 2014.

43. Wu CY, Lin JT, Ho HJ, Su CW, Lee TY, Wang SY, et al. Association of nucleos(t)ide analogue therapy with reduced risk of hepatocellular carcinoma in patients with chronic hepatitis B: a nationwide cohort study. Gastroenterology. 2014;147:143-51.

\section{Submit your next manuscript to BioMed Central and take full advantage of:}

- Convenient online submission

- Thorough peer review

- No space constraints or color figure charges

- Immediate publication on acceptance

- Inclusion in PubMed, CAS, Scopus and Google Scholar

- Research which is freely available for redistribution 